\title{
Simulation and laser vibrometry characterization of piezoelectric AIN thin films
}

\author{
J. Hernando, ${ }^{1, a)}$ J. L. Sánchez-Rojas, ${ }^{1}$ S. González-Castilla, ${ }^{2}$ E. Iborra, ${ }^{2}$ A. Ababneh, ${ }^{3}$ \\ and U. Schmid ${ }^{3}$ \\ ${ }_{1}^{1}$ Departamento Ingeniería Eléctrica, Electrónica, Automática y Comunicaciones, E.T.S.I. Industriales, \\ Universidad de Castilla-La Mancha, 13071 Ciudad Real, Spain \\ ${ }^{2}$ Departamento Tecnología Electrónica, E.T.S.I. Telecomunicaciones, Universidad Politécnica de Madrid, \\ 28040 Madrid, Spain \\ ${ }^{3}$ Chair of Micromechanics, Microfluidics/Microactuators, Faculty of Natural Sciences and Technology II, \\ Saarland University, D-66123 Saarbruecken, Germany
}

(Received 12 March 2008; accepted 18 May 2008; published online 2 September 2008; publisher error corrected 10 September 2008)

In this paper, the electric field induced deformations of sputter-deposited piezoelectric aluminum nitride thin films sandwiched between electrodes on top of a silicon substrate are studied by numerical calculations and scanning laser interferometric measurements. In our calculations based on the finite element method, the results show the displacement of the top and bottom surfaces of both the thin film and the substrate, for either a free or a perfectly clamped structure. The confirmation that the bottom surface of the film is deformed reveals the limitations of techniques that only access the top surface, as well as the double-beam interferometric configuration, under specific conditions. In addition, the simulations demonstrate the dependence of the displacements on the size of the upper electrode and the contribution of the transverse piezoelectric coefficient $d_{31}$ to the features of the displacement profiles. A laser scanning vibrometry technique was used to measure deformations on the top surface with subpicometer vertical resolution. By comparing the calculated and the experimental displacement profiles, an advanced approach is discussed to obtain accurate quantitative information of both coefficients $d_{31}$ and $d_{33}$. (C) 2008 American Institute of Physics. [DOI: 10.1063/1.2957081]

\section{INTRODUCTION}

Many acoustic wave devices and microelectromechanical systems (MEMS) use different piezoelectric thin films as actuating elements. The fabrication of these devices on conventional silicon wafers is becoming increasingly common, due to the possibility of integration into mature silicon technologies. ${ }^{1-3}$ Among the different piezoelectric materials, AlN is the candidate that fulfills most of the requirements for this integration process. Its good mechanical, chemical, and dielectric properties, as well as the compatibility with complementary metal-oxide semiconductor technology, have determined its choice for academic research ${ }^{4,5}$ and even device production in companies such as Avago, Epcos, Infineon, and Trikin.

The assessment of the morphological properties of the piezoelectric films (i.e., composition, crystal quality, residual stress, etc.) is straightforward. However, more complex techniques are required to determine their piezoelectric properties, such as the longitudinal piezoelectric coefficient $d_{33}$. One of the most commonly used techniques to evaluate the goodness of a piezoelectric layer is to obtain the electromechanical coupling factor from acoustic, surface acoustic wave, ${ }^{6}$ and bulk acoustic wave ${ }^{7}$ devices. This factor, in addition to the piezoelectric coefficient $d_{33}$, includes other material properties such as the elastic constant and the dielectric

${ }^{a)}$ Electronic mail: jorge.hernando@uclm.es. permittivity. Therefore, if the material properties are not well known, they will not provide accurate values for the coefficient $d_{33}$. A better approach to determine this coefficient is the direct measurement of the converse piezoelectric effect, that is, the measurement of the strain generated by the application of a known electric field. This is usually accomplished by measuring the thickness variation of a piezoelectric thin film embedded between electrodes on top of a support substrate. This measurement requires resolution in the picometer range in the vertical direction and hence techniques such as atomic force microscopy ${ }^{8,9}$ (AFM) and laser interferometry ${ }^{10-14}$ were applied for this purpose.

The use of a structure consisting of a thin film between electrodes on a substrate has to keep the two following considerations in mind. First, the bending of the substrate, an issue thoroughly covered in the literature. ${ }^{10}$ Due to the actuation of the transverse piezoelectric effect described by the coefficient $d_{31}$, the thin film suffers an in-plane stress that causes the bending of the complete substrate, whether the structure is free or its back surface is not ideally clamped to a rigid holder. This bending superimposes to the piezoelectric thin film thickness variation. The common approach to account for this effect is the use of a double-beam laser interferometry configuration, ${ }^{10,15}$ in such a way that the displacement of both the top and the back surfaces of the structure are monitored simultaneously and the difference between these displacements is assumed to be the thin film thickness variation. Alternatively, instead of accounting for 
it, the substrate bending effect can be minimized by decreasing the upper electrode and/or by gluing the back side of the substrate to a rigid holder. ${ }^{10,13}$

Second, the most important consideration to take into account for this type of structure is that either the tension or the compression of the piezoelectric thin film may give rise to a displacement of both the top free surface of the film and the bottom surface of the film, constrained due to the presence of the deformable substrate. Therefore, the piezoelectric thin film thickness variation distributes between both surfaces. The techniques mentioned before (i.e., AFM and laser interferometry) only access the top surface of the film. Moreover, unfortunately, there is no experimental access to the behavior of the corresponding bottom face. Most of the references in literature that used the two previous techniques either forget or disregard the displacement of the bottom surface of the film for the determination of the piezoelectric coefficients, with the corresponding error. To the best of the authors' knowledge, only the works by Wang et al. ${ }^{16,17}$ on lead-zirconate-titanate (PZT) sandwich structures point out the displacement of the bottom surface of the film. These references, with the help of a finite element model (FEM), report the displacement profile of the top and bottom surfaces of the film, as well as the dependence of these profiles on the size of the upper electrode, for a perfectly clamped structure. Once this consideration is taken into account, the next difficulty is how to estimate the thin film thickness variation from the experimental displacement of the top surface of the film, and how to relate this estimated variation to the piezoelectric coefficient $d_{33}$.

In this work, the piezoelectric performance of sputterdeposited AIN thin films on thick silicon substrate was simulated by FEM analysis and characterized by scanning laser vibrometry. The simulation results show the displacement profile of the top and bottom surfaces of the thin film, as well as the displacement of the top and bottom surfaces of the substrate, for a free and a perfectly clamped structure. The dependence of these displacements on the top electrode size was also calculated. These results emphasize the importance of the deformation of the bottom surface of the film for both the single-beam and the double-beam interferometer configurations. Besides, different characteristics of the displacement profiles were related to the transversal piezoelectric coefficient $d_{31}$. From an experimental point of view, the scanning laser vibrometer data supported the simulated results with a good overall matching between the experimental and the calculated displacement profile of the top surface of the structure. In order to take advantage of the agreement between the experiment and the simulation along this profile, a fitting procedure was applied in order to evaluate the piezoelectric coefficients $d_{33}$ and $d_{31}$ accurately.

\section{METHODS}

\section{A. Device structure}

Figure 1 shows a schematic cross section of the metalpiezo-metal sandwich model for the simulation section. The thickness of the AIN layer and Si substrate is 2 and $640 \mu \mathrm{m}$, respectively. The material of the electrodes is molybdenum

\section{Mo top electrode}

$2 \mu \mathrm{m}$ AlN film

Mo bottom electrode

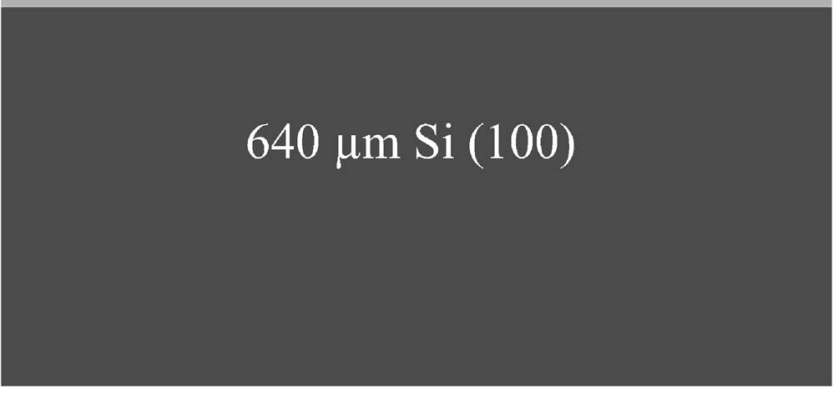

FIG. 1. (Color online) Schematic cross sectional view of the structure for the simulation.

(Mo) with a thickness of $0.2 \mu \mathrm{m}$ for both electrodes. For the experimental results, the main structure of the samples is similar to Fig. 1. The procedure for the fabrication of the samples was the following. First a plain bottom electrode of Mo was deposited on a $640 \mu \mathrm{m}$ thick $\mathrm{Si}(100)$ substrate. Next the AlN thin film was deposited by reactive magnetron sputtering. This film has a pure $c$-axis orientation with a full width at half maximum of the rocking curve around the $\langle 00 \cdot 2\rangle$ direction below $2.5^{\circ}$. The thickness of the AlN film varied from 1.8 to $2 \mu \mathrm{m}$ without appreciable differences in the results shown below. Finally, a top electrode of Mo was defined by photolithography. Circle- and square-shaped top electrodes with different sizes were used. The squares ranged from $100 \times 100$ to $650 \times 650 \mu \mathrm{m}^{2}$ and the circles' diameter ranged from 0.6 to $4 \mathrm{~mm}$. The electrical access to the bottom electrode was made via holes on the AIN layer using wet $\mathrm{KOH}$ etching. For the comparison between experiment and simulation, the exact structure of the sample was implemented in a FEM-based simulation tool.

\section{B. Simulations}

An accurate static computational FEM was built using COVENTORWARE, a commercially available software tool developed for MEMS design. ${ }^{18}$ The mesh used in the model is composed of bricks with orthogonal geometry. The mesh is refined so as to produce accurate results in all the layers, with negligible changes when additional nodes are introduced. In the simulation section, the elastic, piezoelectric, and dielectric constants of AlN are taken from Ref. 19. In particular, the nominal piezoelectric constants are $d_{33}=5.5, d_{31}=-2.6$, and $d_{15}=4 \mathrm{pm} / \mathrm{V}$. These values were modified when the experimental data were fitted to the numerical calculations. Two types of mechanical boundary conditions are considered for the substrate, either free or perfectly clamped. For the clamped structure, the bottom surface of the substrate is assumed to be perfectly fixed in all degrees of freedom. 


\section{Experimental procedure}

The experimental electric field induced out-of-plane displacements were measured with a MSV 400 Polytec scanning laser Doppler vibrometer. The sample was placed on a probe station and electrical contacts to the top and bottom electrodes of the structure were made using tungsten dc tips. The system can scan a certain area of the device. The area is defined by a grid of points and the laser moves from point to point. For each point, the vibrometer obtains a time-domain vibration signal, which is Fourier-transformed to the frequency domain. Therefore, for each point, the amplitude at different frequencies can be obtained, as well as the phase correlation with the other points. All this information is used to obtain an animation of the scanned area movement at different frequencies, as well as displacement profiles along the area.

In this work, the scanned area included the upper electrode as well as the surroundings. The density of points within the grid was limited by the size of the laser spot (i.e., about $5 \mu \mathrm{m}$ ). Points laying exactly at the edges of the electrode were removed from the grid, in order to avoid light scattering effects that could lead to erroneous data. Regarding the boundary conditions of the structure, two different conditions were considered. Either the structure was free on the supporting chuck of the probe station, or the structure was clamped to a $3 \mathrm{~mm}$ thick Al plate by means of different glues, from the standard Loctite Superglue to structural adhesives with Young's modulus as high as $6 \mathrm{GPa}$, such as Loctite 9492. The results for the clamped samples were the same, regardless of the glue used.

For measuring the surface out-of-plane movements, the structure was excited by applying a sinusoidal signal between the electrodes with a given frequency. The amplitude of the applied sinusoidal signal was $17.6 \mathrm{~V}$ (nominally $18 \mathrm{~V}$, reduced by slight loading effects) in all the cases presented in this work. The frequency of the sinusoidal signal must be low enough and far enough from any mechanical resonance so that the experiment can be compared to the static model for a given voltage amplitude.

In order to look for this frequency unaffected by any resonance of the structure, preliminary measurements studied the frequency response of each structure from 10 to $100 \mathrm{kHz}$. The system does not allow to measure below $10 \mathrm{kHz}$ for the conditions required in this work. For this study, a periodic chirp signal was applied between the electrodes. This signal consists of a superposition of sinusoidal signals designed to keep constant the amplitude in the frequency domain and hence allow for a uniform excitation. In the case of the glued structures, for a top electrode with a side either equal to or lower than $200 \mu \mathrm{m}$, the clamping eliminated all the resonances in the $10-100 \mathrm{kHz}$ frequency spectrum, no matter the type of glue used. For larger electrodes, different resonances were found. This effect of the clamping was reported before. ${ }^{13}$ In this paper, the experimental results that are compared to the simulations correspond to glued structures with $200 \mu \mathrm{m}$ square-shaped upper electrodes.
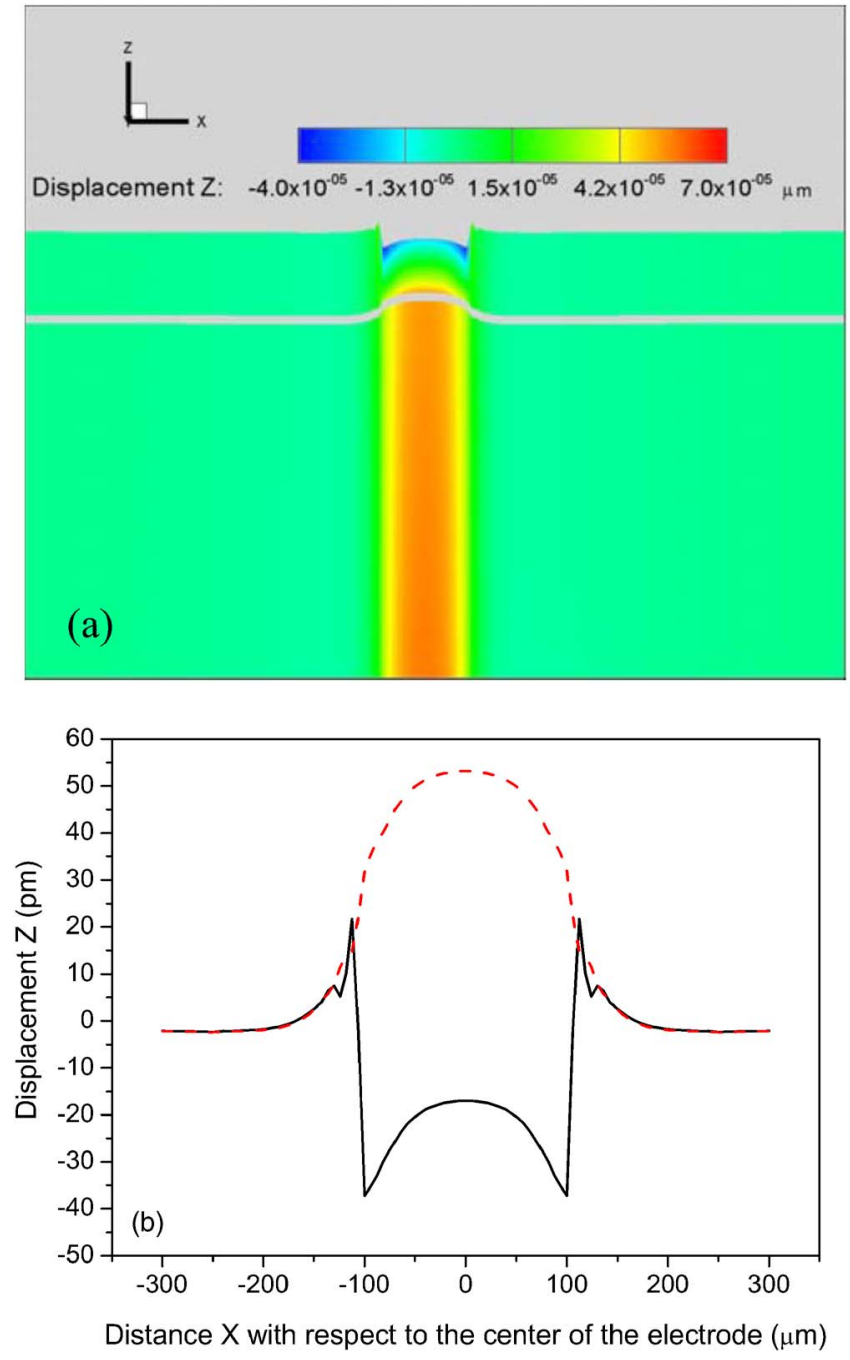

FIG. 2. (Color online) FEM results for Fig. 1 with the following conditions: the side of the square top electrode is $200 \mu \mathrm{m}$, the applied voltage is $17.6 \mathrm{~V}$, and the boundary condition is a perfectly clamped bottom surface of the substrate. (a) Cross sectional view perpendicular to the top electrode surface. The vertical displacement along the $Z$ axis is magnified for a better view. (b) $Z$ displacement profiles of the top (solid curve) and the bottom (dashed curve) surfaces of the thin film against the $X$ in plane coordinate, whose origin is taken at the center of the top electrode $(X=0)$. The origin of the vertical axis corresponds to the situation of no deformation for the profile under consideration.

\section{RESULTS AND DISCUSSIONS}

\section{A. Simulation results}

First we present the results of the FEM calculation for the structure of Fig. 1 with a perfectly clamped bottom face of the substrate. Figure 2 shows the results for a $200 \mu \mathrm{m}$ square-shaped electrode and an applied voltage of $17.6 \mathrm{~V}$. Figure 2(a) shows a cross sectional view perpendicular to the top electrode surface and along its center. The $X, Y$, and $Z$ axes are plotted for orientation purposes. Only the $2 \mu \mathrm{m}$ thick piezoelectric layer and a part of the $640 \mu \mathrm{m}$ thick substrate are represented. Both electrodes are removed as they follow the deformation of the adjacent layers. The vertical displacement along the $Z$ axis is magnified for a better view. The color is also modulated following the vertical displacement. The $Z$ displacement color scale is in microns. A 
quantitative representation is shown in Fig. 2(b), where the $Z$ displacement profiles of the top (solid) and the bottom (dashed) surfaces of the thin film are plotted against the $X$ in-plane coordinate, whose origin is taken at the center of the top electrode $(X=0)$. The origin of the vertical axis in this figure corresponds to the situation of no deformation for the curve under consideration.

The most important point to notice from this figure is the displacement of the bottom surface of the film. This displacement is far from being negligible, with a value of $53 \mathrm{pm}$ at the center of the electrode. For comparison, the displacement on the top surface is $-17 \mathrm{pm}$ at the same place. Therefore, for this electrode size, the total change in thickness in the piezoelectric layer is distributed at the top and the bottom surfaces. This fact brings out very serious limitations to the determination of the piezoelectric coefficient $d_{33}$ from a measurement of the displacement of the top surface of the structure, as it is the case with laser scanning interferometry and AFM. In the way Fig. 2(b) is presented, the electric field induced change in the film thickness at the center of the electrode is directly given by the separation between the two curves at that point. Besides, the displacement of the bottom surface of the film is equivalent to the displacement of the top surface of the substrate. Consequently, there is a significant change in the substrate thickness below the top electrode. This would not occur at a perfectly rigid substrate. It is also worth mentioning that there is no bending of the substrate due to the boundary condition of perfect clamping.

As the top electrode size increases, the displacement of the top surface of the film at the center of the electrode increases, while the displacement of the bottom surface of the film decreases. This correlation is valid up to a critical electrode size, which is around $2 \mathrm{~mm}$ for a $2 \mu \mathrm{m}$ thick AlN film. Beyond this critical size, the bottom displacement measured at the center of the electrode is negligible. The top displacement saturates and corresponds directly to the film thickness variation. This behavior was already predicted by Wang et al. ${ }^{16}$ in a perfectly clamped structure made of PZT. This result suggests the suitability of electrodes with a size larger than the critical one when techniques are used that only access the top surface of the structure. We will come back to this point in Sec. III B.

Figure 2 also shows the profile of the top surface of the film. This profile is characterized by different features around the edges of the top electrode. These features are the step in the displacement at the edges of the electrode and the nonuniform displacement at both sides of the step. The curvature of the top surface of the film below the top electrode is a result of the nonuniform displacement at the sides of the edges below the top electrode.

Other important effects, evidenced by the simulations, are illustrated through Fig. 3, where we assume exactly the same model as for Fig. 2, except for $d_{31}$, which is fixed at 0 . First, while in Fig. 2 there is a displacement of the bottom surface of the film, in this case the film compression is only accommodated on the top surface of the film, with no change at the bottom face of the film. The magnitude of the film thickness variation is given exactly by the product of the coefficient $d_{33}$ and the applied voltage, as if the film was
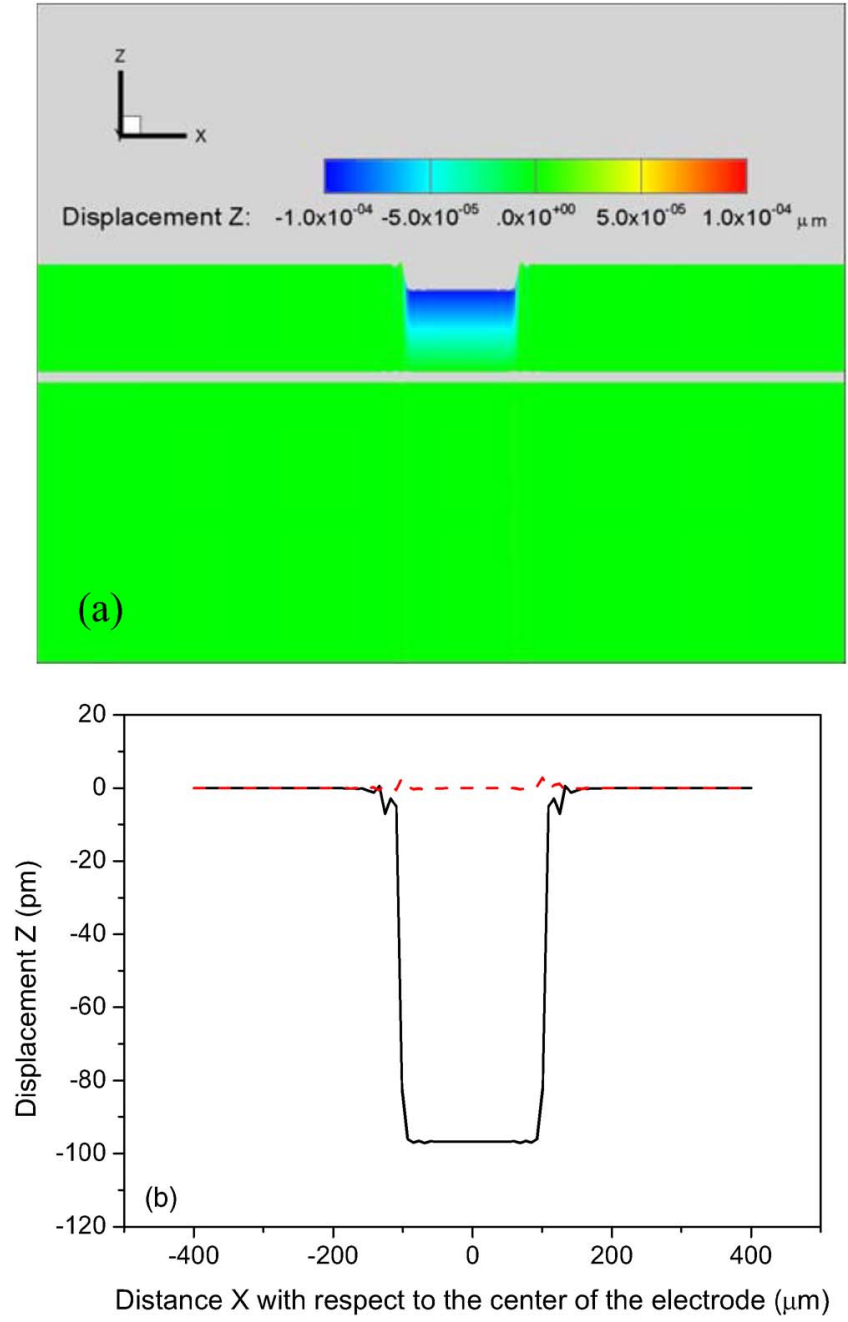

FIG. 3. (Color online) Equivalent to Fig. 2, but $d_{31}=0 \mathrm{pm} / \mathrm{V}$.

freestanding. Second, Fig. 3(b) shows that the displacement of the top surface of the thin film below the area covered by the top electrode is plain, while in Fig. 2 this displacement is characterized by the curvature mentioned before. In addition, the film thickness variation at the center of the electrode changes from $70 \mathrm{pm}$ in Fig. 2 to $97 \mathrm{pm}$ in Fig. 3. Therefore, the conclusion of this numerical change in $d_{31}$ is that this coefficient plays an important role in the profile of the displacement of the two film surfaces.

Our model was also used to analyze the case of a free structure. Figure 4 shows the same information as in Fig. 2 for the same structure and conditions, but the substrate is free. Part (b) shows the displacement of three surfaces: the top (solid) and bottom (dashed) surfaces of the film, and the bottom surface of the substrate (dotted). As mentioned before, the displacement of the bottom surface of the film is equivalent to the displacement of the top surface of the substrate. The displacement 0 corresponds to the situation of no deformation for the surface under consideration. From these figures, the expected overall bending of the substrate, which was taken into account previously by different authors, ${ }^{10,13}$ can clearly be seen. In part (a), the bending can be noticed outside the top electrode, since the further from the electrode, the smaller the displacement of the layers. Also, in part 

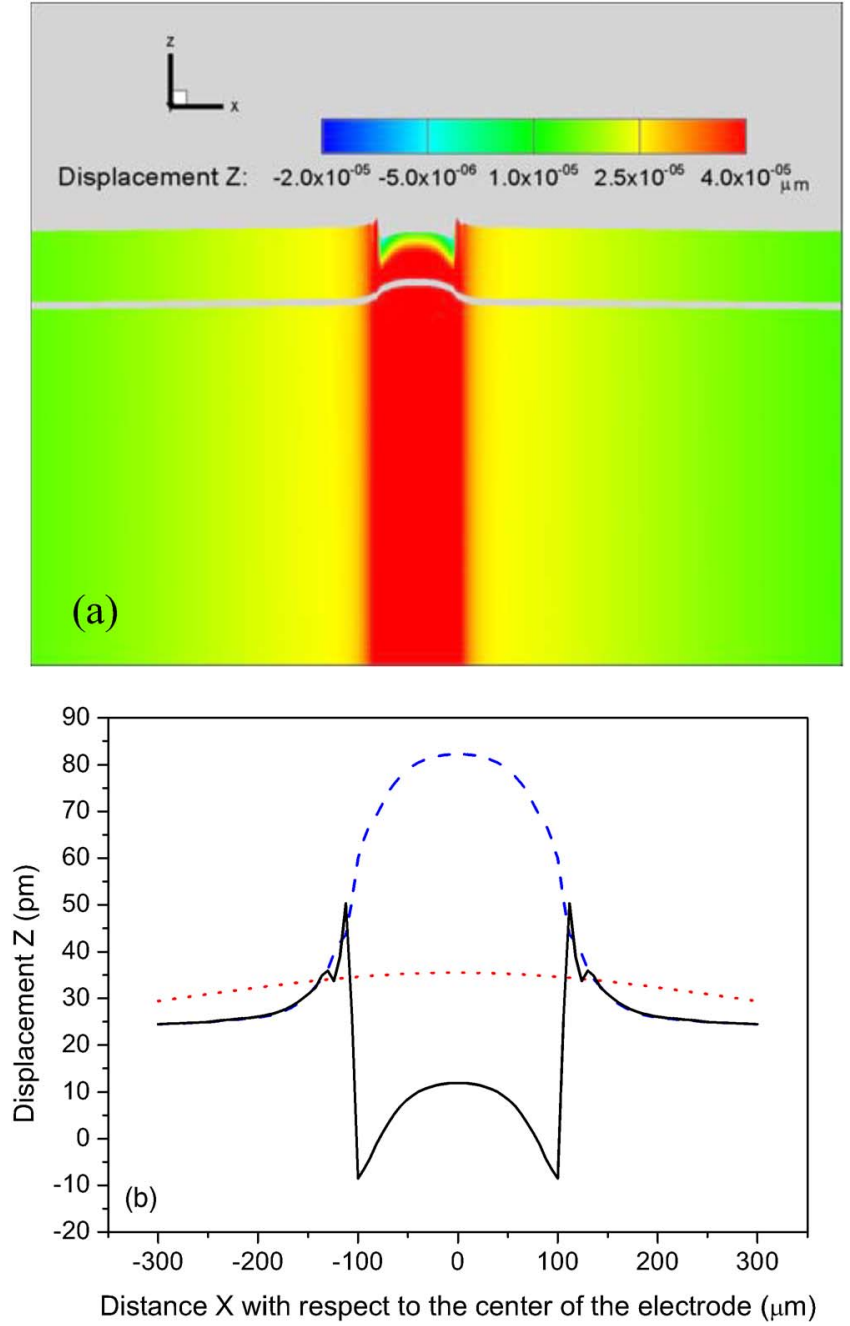

FIG. 4. (Color online) Equivalent to Fig. 2, but the structure is free. Part (b) includes the displacement of the bottom surface of the substrate (dotted curve).

(b), the deformation of the bottom surface of the substrate confirms the bending. It is worth mentioning that, for a positive voltage, the coefficient $d_{33}$ causes a compression of the piezoelectric layer in the $Z$ direction (i.e., negative displacement for the top surface), while the coefficient $d_{31}$, opposite sign to $d_{33}$, gives rise to a tension of the piezoelectric layer in the $X$ direction. This last tension results in the bending of the substrate with a positive displacement along the $Z$ direction. In addition to this bending, the results show, as in the case of the perfectly clamped structure, the deformation of the bottom surface of the piezoelectric thin film, and correspondingly the deformation of the top surface of the substrate.

It can be noticed that the displacements at the top and bottom faces of the substrate follow a different profile and, in consequence, there is also a change in the substrate thickness over the region covered by the top electrode with respect to the regions outside the top electrode. This is a crucial result for the interpretation of double-beam interferometer measurements with laser beams reflected on the top electrode and the back side of the substrate. With this approach, the thickness variation of the piezoelectric film is assumed to be equal to the difference between the displacement of the bottom surface of the substrate and the displacement of the top surface of the structure. This would be correct if the displacement of the top and bottom surfaces of the substrate are equal, i.e., the thickness of the substrate remains constant. However, as it can be seen in Fig. 4, this may not be the case due to the deformation of the piezoelectric thin film. The associated error depends on the exact geometry of the layers and the upper electrode area. Specifically, in the case of a $200 \mu \mathrm{m}$ side square electrode, the difference between the displacement of the bottom surface of the substrate and the displacement of the top surface of the film at the center of the electrode is $23 \mathrm{pm}$, while the difference between the displacements of the top and bottom surfaces of the film, the correct value, is $70 \mathrm{pm}$. For a $1 \mathrm{~mm}$ side square electrode, these values are 100 and $70 \mathrm{pm}$, respectively. So, the error is lower for larger electrode.

\section{B. Experimental results}

The aim of this section is to confirm the simulation results experimentally and to obtain an accurate value for the piezoelectric coefficients $d_{33}$ and $d_{31}$ of AlN films. The scanning laser vibrometer was used to obtain an animation of the movement of a scanned area that included the top electrode and the surroundings of a structure glued to an Al holder in an attempt to clamp the bottom surface of the substrate, with a $2 \mu \mathrm{m}$ thick AlN film and a $200 \times 200 \mu \mathrm{m}^{2}$ square top electrode (this animation is video $1,{ }^{20}$ where the black grid corresponds to null displacement). Figure 5 shows the displacements obtained from the previous animation at (a) 17.6 and (b) $-17.6 \mathrm{~V}$. The top electrode can be seen as well as the tip contacting it. The parameters of the measurements were given in Sec. II C. In this case, the frequency of the sinusoidal signal was $60 \mathrm{kHz}$, far from any mechanical resonance. It is also illustrative to see an animation of the movement of the profile along a line that crosses the top electrode from left to right (video $2,{ }^{20}$ where the upper part shows a top view of the structure as well as the line along which the displacement is measured, and the lower part shows the animation along that line). Figure 6 shows the displacement profiles obtained from video 2 at (a) 17.6 and (b) $-17.6 \mathrm{~V}$. Figures 7 and 8 are equivalent to Figs. 5 and 6, respectively, but the same structure is free (videos 3 and $4^{20}$ are equivalent to videos 1 and 2, respectively, but the same structure is free). Certain noise is present in the measurements due to the fact that the data are not smoothed by any filtering.

It is worth pointing out that the features of the displacement of the top surface of the film around the edges of the top electrode, predicted by the FEM in Fig. 2, are clearly detected in the experiment. Therefore, our measurements support the predictions deduced by the numerical calculations for the top surface of the film, and this would indirectly confirm the deformation of the bottom surface of the film.

These measurements also show the predicted bending of the substrate. It can be clearly seen in Figs. 7 and 8 for the free structure, since there is a noticeable displacement of the structure outside of the top electrode. The bending is also present for the glued structure in Figs. 5 and 6, although to a lower extent due to the gluing. Incidentally, it can be noticed 

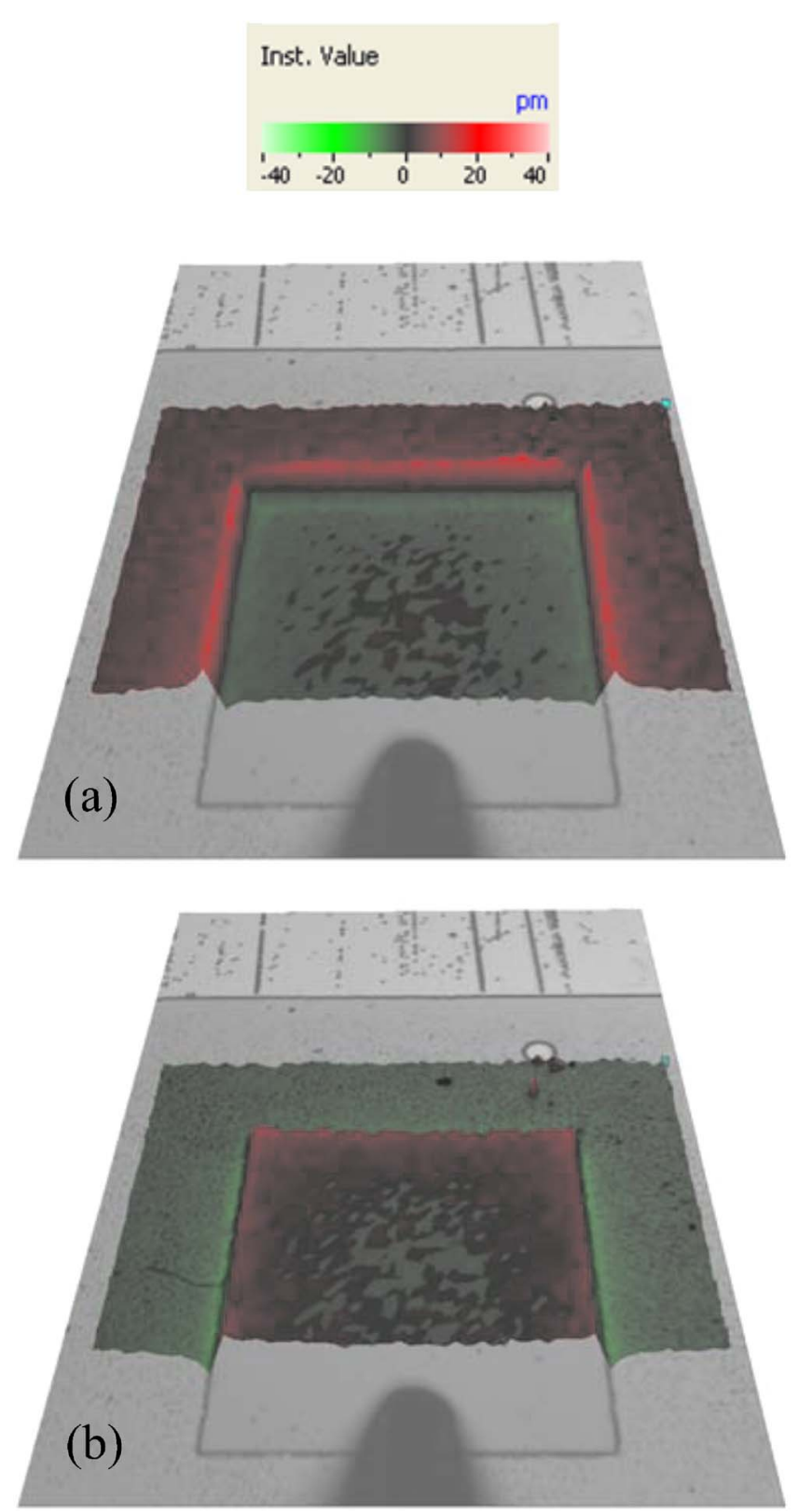

FIG. 5. (Color online) Scanning laser vibrometer measured displacement for a $2 \mu \mathrm{m}$ AlN glued structure over an area covering part of a $200 \mu \mathrm{m}$ side square electrode and the surroundings at (a) 17.6 and (b) $-17.6 \mathrm{~V}$.

that the displacement of the central area of the top electrode is almost zero during the whole animation. This is only possible if the magnitude of the displacement due to the bending is equal to the magnitude of the displacement due to the piezoelectric actuation. As mentioned before, these displacements are of opposite sign and they may cancel each other. This conclusion was checked by using electrodes of smaller and bigger sizes. The bigger the electrode, the larger the bending. And the shift of the animation of the displacement along the $Z$ axis, for the different electrode sizes, corresponded to the expected bending change. It can also be mentioned that the bending is expected to be constant over the area of the $200 \mu \mathrm{m}$ side square electrode. From these results, we can conclude that our conditions for the clamping of this structure to a holder by means of the glue do not reproduce the ideal clamping boundary conditions used for
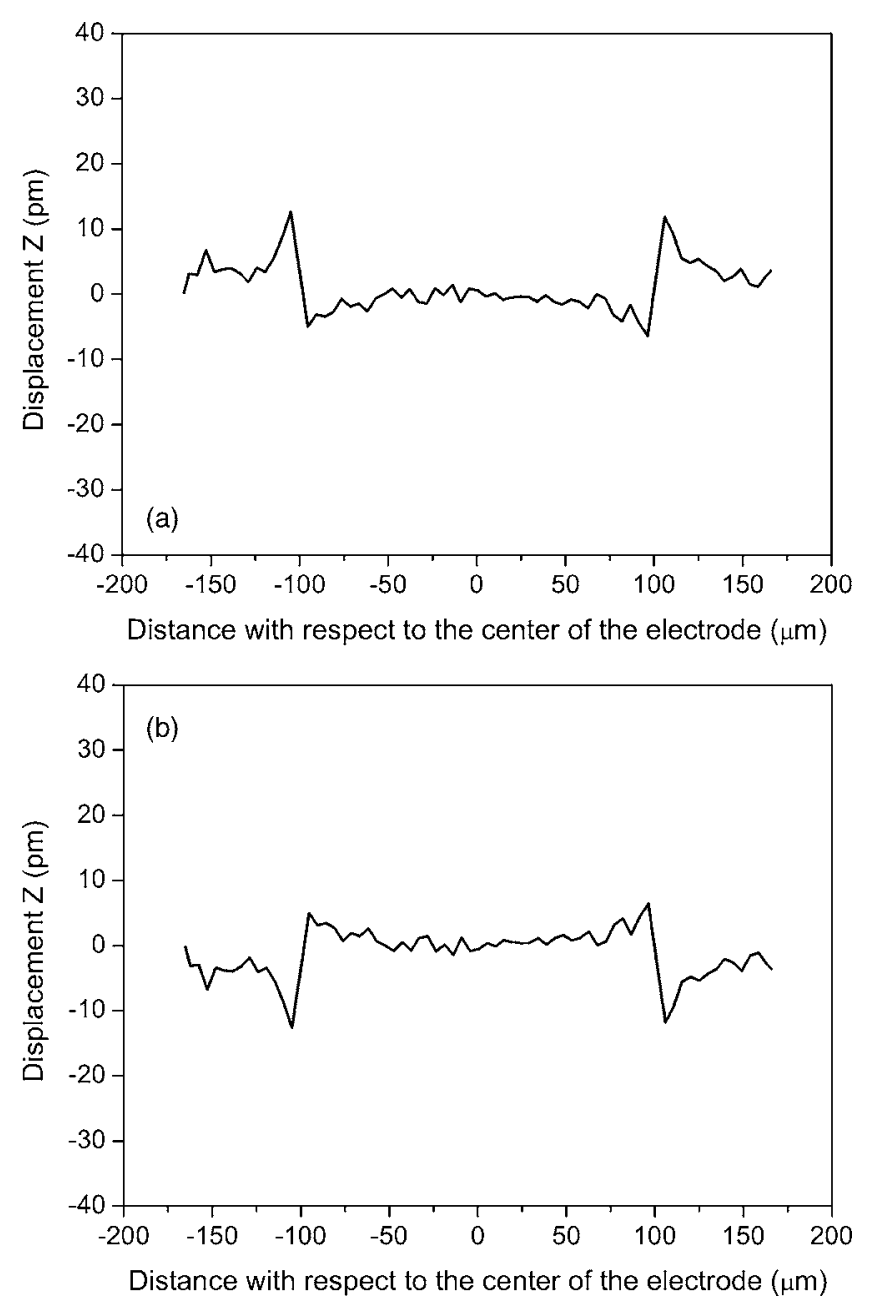

FIG. 6. Scanning laser vibrometer measured displacement profile for a $2 \mu \mathrm{m}$ AlN glued structure along a line that crosses a $200 \mu \mathrm{m}$ side square electrode and the surroundings at (a) 17.6 and (b) $-17.6 \mathrm{~V}$.

the simulations. There is a bending due to the compliance of the glue. All these results show that the displacement of the top surface of the structure might have a contribution due to the bending and the magnitude of this contribution depends on parameters such as the size of the electrode and the consistency of the clamping.

Next, our goal is to estimate the magnitude of the piezoelectric coefficients based on all the computed and measured results described above. To this end, several approaches were considered. According to the simulations, the displacement of the top surface of perfectly clamped AIN structures saturates as the electrode area increases, reaching the total film thickness variation at the center of the top electrode for electrodes with a size larger than $2 \mathrm{~mm}$. By contrast, even for square electrodes with a side as low as $400 \mu \mathrm{m}$, multiple resonances appear experimentally; and for electrodes with a diameter equal or greater than $2 \mathrm{~mm}$, the frequency response was dominated by many mechanical resonances, despite the gluing with very stiff adhesives. As a consequence, when glued structures, with such large electrodes, were excited by applying a sinusoidal signal at a frequency outside the resonances, the experimental displacement was dominated by the influence of the resonances and the substrate bending, and no 

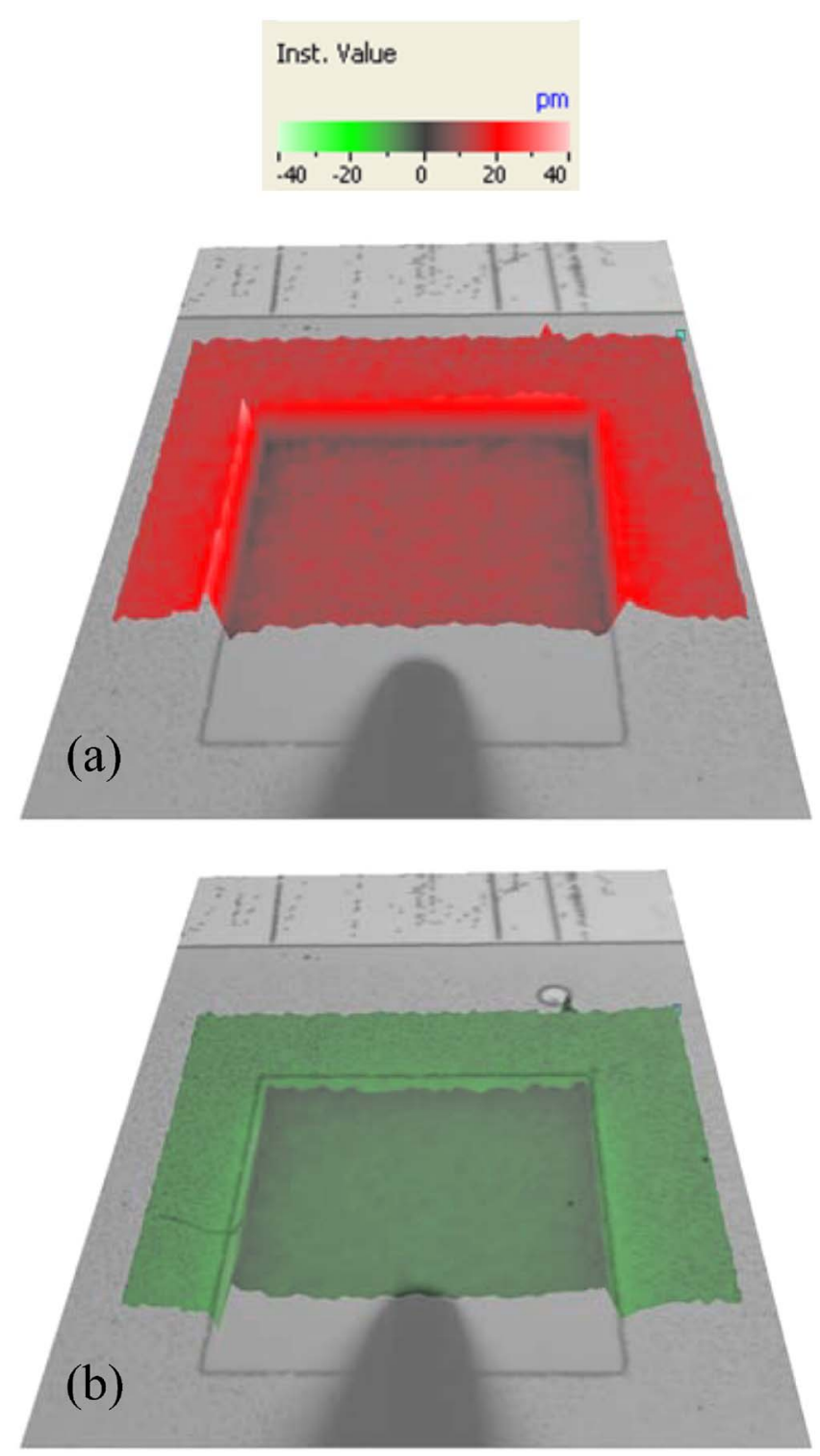

FIG. 7. (Color online) Equivalent to Fig. 5, but the same structure is free.

evidence of the expected saturation was detected. This approach was then discarded due to the difficulties in achieving the perfect clamping condition.

Another approach to obtain the coefficient $d_{33}$ from scanning laser vibrometer measurements is to use the magnitude of the step at the edges of the top electrode. ${ }^{17,21}$ This procedure is based on the approximation that this step equals the thin film thickness variation, what depends on the size of the electrode. For example, this is not the case in Fig. 2. In addition, the magnitude of this step has to be associated with $d_{33}$. The usual procedure is to relate it to an effective $d_{33}$, assuming that the substrate is perfectly rigid. ${ }^{22}$ However, from our results, it is clear that the substrate deforms, which is what limits the applicability of this approach.

For all these reasons, we propose the following approach. As the displacement profile measured experimentally resembles very well all the features of the simulated profile, it would be reasonable to obtain the piezoelectric coefficients by fitting the simulated profile to the experimental one. In this way, the whole profile, and not only a particular feature
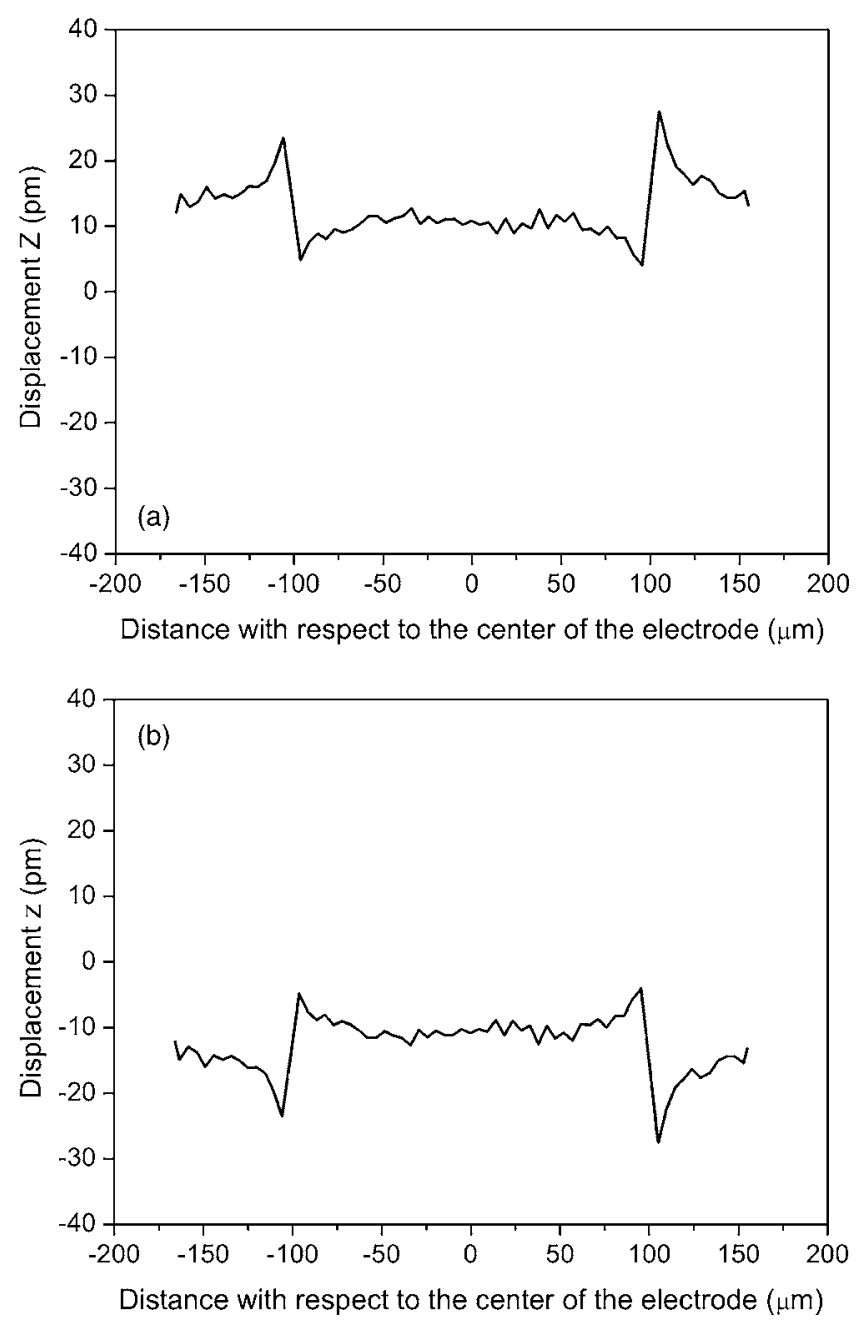

FIG. 8. Equivalent to Fig. 6, but the same structure is free.

such as the step, would be used for the evaluation of the piezoelectric coefficients. The measured profile must not be affected by any resonance of the structure in the tens of kilohertz range. According to the previous sections, this implies the use of top electrodes with a side of $200 \mu \mathrm{m}$ or smaller, as well as gluing the structure to a holder. The results obtained under these conditions can be compared to the simulations for a perfectly clamped structure.

Figure 9 shows a fitting example. The solid curve is an average of the measured profiles over a $200 \times 200 \mu \mathrm{m}^{2}$ square top electrode for a glued, $1.8 \mu \mathrm{m}$ thick, AlN structure. The sinusoidal excitation signal had an amplitude of $17.6 \mathrm{~V}$ at $60 \mathrm{kHz}$. This curve is characteristic of the many different measurements performed in different samples. Two calculated displacement profiles are also included in the figure, associated with two sets of piezoelectric coefficients. The dotted curve corresponds to the nominal values for $d_{i j}$ (see Sec. II B). In this case, the measured curve is less pronounced and has lower vertical displacements than predicted by the model. Next, we tried to find the values of $d_{33}$ and $d_{31}$ that best fit the experimental curve for this sample; in this process we found out that the main features of the profile are (i) the magnitude of the step and (ii) the total deformation of the top surface of the film under the electrode, going from one edge to the center. These two degrees of freedom allow 


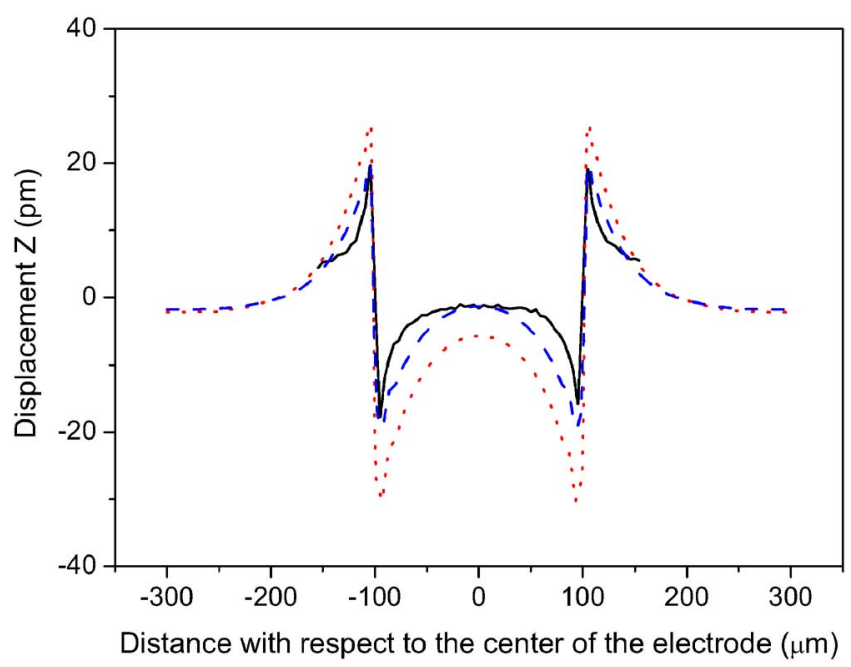

FIG. 9. (Color online) Fitting to the experimental result with the numerical calculations. The solid line corresponds to the average of different measured displacement profiles over a $200 \mu \mathrm{m}$ side square top electrode for a $1.8 \mu \mathrm{m}$ AlN glued structure. The dotted line is the calculated result for the nominal $d_{i j}$ specified in Sec. II B. The dashed line is the simulation for $d_{33}=4 \mathrm{pm} / \mathrm{V}$ and $d_{31}=-2 \mathrm{pm} / \mathrm{V}$.

to vary both $d_{33}$ and $d_{31}$ independently. We found a good agreement with the dashed curve, which corresponds to $d_{33}$ $=4 \mathrm{pm} / \mathrm{V}$ and $d_{31}=-2 \mathrm{pm} / \mathrm{V}$. With these values, the step at the edges of the top electrode and the curvature under the top electrode are comparable for both the experiment and the simulation. In this way, the estimated piezoelectric coefficients are deduced from a comparison between experiment and theory that takes into account the whole displacement profile, and not only a particular point or feature in the measurement.

Regarding the accuracy of the procedure for the different samples measured, the standard deviation of the displacement obtained from the averaged profiles is below $\pm 2 \mathrm{pm}$. This error has to be extrapolated to the value of the piezoelectric coefficients. According to our FEM analysis, a variation of $2 \mathrm{pm}$ in the main features of the experimental profile of Fig. 9 corresponds to a variation of $3 \%$ and $10 \%$ in the value of $d_{33}$ and $d_{31}$, respectively, i.e., $d_{33}=4 \pm 0.1 \mathrm{pm} / \mathrm{V}$ and $d_{31}=-2 \pm 0.2 \mathrm{pm} / \mathrm{V}$.

Besides, a number of material constants are assumed in the fitting process, being the AlN elastic coefficients the most critical, since the magnitude of these coefficients is not very well established in the literature. For example, the value of $C_{11}$ used in this work and obtained from Ref. 19 is $345 \mathrm{GPa}$, while other publications reported a value of $411 \mathrm{GPa}^{23}$ This is a variation close to $20 \%$. This uncertainty in the magnitude of $C_{11}$ results in another source of error. Depending on the elastic constant used between the two extremes above, we estimated a variation of $8 \%$ in the magnitude of $d_{33}$ and a variation of $12 \%$ in the value of $d_{31}$ when fitting the experimental profile of Fig. 9. It is worth to remark that this error is not intrinsic to our procedure, but has to be attributed to the inaccuracy of the elastic constants of AlN.

The comparison of our results with other publications cannot consider those publications that do not include the deformation of the bottom surface of the piezoelectric layer to determine the piezoelectric coefficients. An appropriate comparison can be made with Ref. 19, where the phase velocity of AlN-based surface acoustic wave devices, grown by metal organic chemical vapor deposition (MOCVD), was used to estimate the magnitude of the different parameters of AlN. According to this reference, $d_{33}=5.5 \mathrm{pm} / \mathrm{V}$ and $d_{31}=$ $-2.6 \mathrm{pm} / \mathrm{V}$. These values are higher than those obtained from Fig. 9. Since the accuracy of our procedure is enough to detect this difference, we attribute it to a difference in the AlN crystalline quality when samples are grown by different techniques such as MOCVD and sputtering.

\section{CONCLUSIONS}

AlN piezoelectric thin films embedded between electrodes on top of a silicon substrate were studied using FEM analysis and scanning laser vibrometry, in terms of the displacement generated by the voltage applied between the top and bottom electrodes, with the aim of obtaining accurate values of the piezoelectric coefficients. FEM simulations, which take into account the compliance of the substrate, predict a pronounced deformation of the bottom surface of the thin film located below the top electrode, and correspondingly of the top face of the substrate, for the top electrode sizes commonly used in the literature. Therefore, the thin film thickness variation is thus the result of both the top and bottom surface displacements. Since techniques such as scanning laser vibrometry measure only the displacement on the top surface, the change in film thickness cannot be directly deduced. As a result, the immediate determination of the piezoelectric coefficient $d_{33}$ from the measurement of the displacement of the top surface of the structure is not straightforward. In our case, based on the excellent agreement between the experimental and the calculated displacement profiles, the values of $d_{33}$ and $d_{31}$ in AlN were deduced by applying a fitting procedure that includes the different features of the profile along the top electrode.

\section{ACKNOWLEDGMENTS}

This work was supported by the Junta de Comunidades de Castilla La Mancha under Project No. PCC08-0015-0722 and the Spanish Ministerio de Educación y Ciencia under Project No. DPI2006-01923.

${ }^{1}$ B. Piekarski, D. DeVoe, M. Dubey, R. Kaul, and J. Conrad, Sens. Actuators, A 91, 313 (2001).

${ }^{2}$ G. Perçin and B. T. Khuri-Yakub, Ultrasonics 40, 441 (2002).

${ }^{3}$ P. Cong, T.-L. Ren, and L.-T. Liu, Microelectron. Eng. 66, 779 (2003).

${ }^{4}$ E. Iborra, M. Clement, J. Sangrador, A. Sanz-Hervás, L. Vergara, and M. Aguilar, IEEE Trans. Ultrason. Ferroelectr. Freq. Control 51, 352 (2004).

${ }^{5}$ S. Günthner, M. Egretzberger, A. Kugi, K. Kapser, B. Hartmann, U. Schmid, and H. Seidel, IEEE Sens. J. 6, 596 (2006).

${ }^{6}$ E. Iborra, L. Vergara, J. Sangrador, M. Clement, A. Sanz-Hervás, and J. Olivares, IEEE Trans. Ultrason. Ferroelectr. Freq. Control 54, 2367 (2007).

${ }^{7}$ M.-A. Dubois and P. Muralt, Appl. Phys. Lett. 74, 3032 (1999).

${ }^{8}$ J. A. Christman, R. R. Woolcott, Jr., A. I. Kingon, and R. J. Nemanich, Appl. Phys. Lett. 73, 3851 (1998).

${ }^{9}$ K. Tonisch, V. Cimalla, Ch. Foerster, H. Romanus, O. Ambacher, and D. Donstov, Sens. Actuators, A 132, 658 (2006).

${ }^{10}$ A. L. Kholkin, Ch. Wütchrich, D. V. Taylor, and N. Setter, Rev. Sci. Instrum. 67, 1935 (1996).

${ }^{11}$ M.-A. Dubois and P. Muralt, J. Appl. Phys. 89, 6389 (2001). 
${ }^{12}$ K. Yao and F. E. H. Tay, IEEE Trans. Ultrason. Ferroelectr. Freq. Control 50, 113 (2003).

${ }^{13}$ R. Herdier, D. Jenkins, E. Dogheche, D. Rèmiens, and M. Sulc, Rev. Sci. Instrum. 77, 093905 (2006).

${ }^{14}$ Q. Chen, L. Qin, and Q.-M. Wang, J. Appl. Phys. 101, 084103 (2007).

${ }^{15}$ W. Y. Pan and L. E. Cross, Rev. Sci. Instrum. 60, 2071 (1989).

${ }^{16}$ Z. Wang, G. K. Lau, W. Zhu, and C. Chao, IEEE Trans. Ultrason. Ferroelectr. Freq. Control 53, 15 (2006)

${ }^{17}$ Z. Wang and J. Miao, J. Phys. D 41, 035306 (2008).

${ }^{18}$ Coventor Inc., COVEnTORWARE ${ }^{\mathrm{TM}}$ Reference Guide, Cary, NC, 2002 (www.coventor.com).
${ }^{19}$ K. Tsubouchi and N. Mikoshiba, IEEE Trans. Sonics Ultrason. SU-32, 634 (1985).

${ }^{20}$ See EPAPS Document No. E-JAPIAU-104-149814 for four movie files: video 1 , video 2 , video 3 , and video 4 . Their content is explained in the text. For more information on EPAPS, see http://www.aip.org/pubservs/ epaps.html.

${ }^{21}$ C. Chao, Z. Wang, W. Zhu, and O. Tan, Rev. Sci. Instrum. 76, 063906 (2005).

${ }^{22}$ K. Lefki and G. J. M. Dormans, J. Appl. Phys. 76, 1764 (1994).

${ }^{23}$ L. E. McNeil, M. Grimsditch, and R. H. French, J. Am. Ceram. Soc. 76, 1132 (1993). 